\title{
Research on control method of complex time-varying system
}

\author{
Tian Hongbin \\ Henan Polytechnic \\ Zhenzhou , 450046, China \\ 13938522103@139.com
}

\begin{abstract}
Because the charged objects have the factors of complexity, interference uncertainty and nonlinear, the timevarying system control method gains increasingly attentions. First, this paper carries the researches on the time-varying system classification and builds the control model of the timevarying system. Then the paper discusses the common time varying system control method and analyzes the state-of-art and performance of the control algorithms. Finally, it investigates the development tendency of the complex timevarying system control methods.
\end{abstract}

Keywords-control system; time-varying system; nonlinear; adaptive control; prediction control

\section{INTRODUCTION}

The time-varying systems are widely applied in complex system process control and aerospace technology. According to the different emphases of different fields of time-varying systems, the time-varying systems can be basically classified to a few types. According to system itself moving conditions, there are parameters time-varying, uncertain and construction time-varying. According to the factors causing time-varying, there are system model time-varying and system uncertain disturbance. According to the control technology, there are nonlinear and linear time-varying. Therefore, before researching on the time-varying system, the time-varying system features and types should be deeply analyzed because it directly affects the performance of the control algorithms and the system stability.

The researches on the time-varying system should begin with the time-varying system identification methods, control methods and stable analysis due to the dynamics. The identification of time-varying systems [1] is mainly to suggest the research theories and methods of the corresponding matching time-varying systems based on the system input and output. Generally, it includes on-line identification and off-line identification. Relative to the timevarying feature of the system, the system time-varying identification is usually required to be online which can complete the model with continuous identification when the system model is unknown. In addition, system changes make control model in the system keep refinement and improvement. Thus, the system identification is normally as one part of the control in the time-varying system. The stability of time-varying systems [2] carries researches on the boundaries of the input and output parameters and states with time-varying disturbances which is the basic of the control system. However, the complex factors of the time-varying system will lead the control system stability reduced.
Therefore, while designing the control effects, the timevarying analysis is carried under some conditions.

This paper discusses the time-varying control system research progress by analyzing the time-varying control system modeling and generalizes the practical applications.

\section{Control Model Of The Time-VArying System}

The time-varying model description method currently based on the unknown time-varying system identification with Markov model which can describe the dynamic weight vector of the unknown system and get familiar with the timevarying components of the dynamic weight vector.

\section{A. The system recognition based on Markov model}

This model is the common used dynamic model when study the discrete time-varying conditions which contain one-order Markov process and multiple regressions.

Assuming the weight vector of the dynamic system based on self-adaptive filter can be represented by one-order Markov process.

$$
w_{0}(k+1)=a w_{0}(k)+\omega(k)
$$

In the equation, $a$ is model parameter, $k$ is discrete sampling time, $\omega(k)$ 为 is the process noises vector whose mean is 0 . The weight vector $w_{0}(k)$ can be regarded as the generation factor of process noises $\omega(k)$. After low-pass filtering the components, the low-pass filter transformation function is $1 /\left(1-a z^{-1}\right), z^{-1}$ is delay factor. If $a \rightarrow 1$, the Markov model will drastically change until multiple iterations.

Form multiple regressions, assume the expectation function of the time-varying system is $d(k)$, there is:

$$
d(k)=w_{0}^{H}(k) u(k)+v(k)
$$

In the equation, the measurement noise is $v(k)$ assuming it's the white noise with mean of 0 and variance of $\sigma_{v}^{2}$. Therefore the input vector and noise vector are stable process respectively. However, because $w_{0}(k)$ is timevarying variable, the expectation of the output is still nonstable. 


\section{B. Time-varying control system model}

Assume the time-varying controlled objects can be described as:

$$
\left\{\begin{array}{l}
\dot{x}(t)=f_{1}[x(t), u(t), \theta(t), t] \\
y(t)=f_{2}[x(t), u(t), \theta(t), t]
\end{array}\right.
$$

In the equation, the system states are $x(t)$, system input is $u(t)$, output is $y(t)$, the time-varying parameter is $\theta(t)$.

Discrete the above equations and consider the noises and disturbance, there are:

$$
\left\{\begin{array}{l}
x(k+1)=\Phi(\theta(k), k) x(k)+\Gamma(\theta(k), k) u(k)+\omega(k) \\
y(k)=H(\theta(k), k) x(k)+v(k)
\end{array}\right.
$$

In the equation, the discrete sampling time is $k$, the random disturbance is $\omega(k)$, the measured noise is $v(k)$. $\Phi(\theta(k), k), \Gamma(\theta(k), k)$ and $H(\theta(k), k)$ are $n \times n, n \times m$ and $r \times n$ matrixes respectively.

\section{TIME-VARYING SySTEM CONTROL ANALYSIS}

\section{A. 3.1 The adaptive control of the slow time-varying system}

The self-adaptive control and feedback control are based on model control method as well as the optimal control. The differences are that the system models need to be continuously correct based on online identification aiming at the system uncertainty analysis. The self-adaptive control methods are widely researched in Nineteen Seventies. Reference[3] proposed to introduce the system model reference self-adaptive control parameters of linear system to linear time-varying system which applied modeling error parameters to linearize for system self-adaptive control. There were better control effects but these control methods only dealt with slow time-varying system and the timevarying regions are known. Reference [4] proposed the selfadaptive control algorithm with state gradually advancing models assuming the parameters were unknown and limited. Reference [5] proposed the system with parameter disturbance unknown and designed self-adaptive feedback control algorithm. While tracking the errors, the error can converge to zero, but these algorithms had strict requirements for the time-varying parameter.

\section{B. The robust adaptive control with uncertain disturbance}

Aiming at problems that the system self-adaptive control is restrained by the parameter change factors in the traditional time-varying system, the self-adaptive control with robust is proposed. Reference [6] designed robust selfadaptive filters pointing to the disturbance and time-varying unknown system which took advantage of the parameter signals adjustment to complete the system dynamic performance. Although this algorithm requires very few for the parameters in the time domain, there are strict assumption in other parameters. Reference [7] proposed the robust self-adaptive control based on model references and the applications in linear time-varying system. It obtained some results but the commodity of the method still need some work.

\section{The control methods based on special time-varying}

\section{1) Repetitive learning control}

The repetitive learning control introduces the learning mechanism to the control method to make it have some learning capabilities. Additionally, it input the signal model into system model which lead the system periodically track the inference signals or input. Compared with other control method, this method has better control precisions and widely applied in the server control and high-precision manufacture.

2) Periodical adaptive control

When the periods of the system uncertain parameter are known, the parameter periodic self-adaptive control model of the self-adaptive controller can be constructed by compute point by point in order to make the tracking errors gradually converge which can solve the period self-adaptive control for one-order mixture parameter uncertain system. The reference[10] proposed the self-adaptive period control algorithm. The non-minimum phrase system can obtain better control effects when predict the order information of the controlled objects.

\section{3) Model prediction control}

Because of time-variability characteristic, there is uncertainty when build the mathematical model for the practical objects. Thus it's hard to design controller when the order information is quite high. Aiming at this problem, the predictive control began to develop. The model prediction model proposed in reference [11] was applied in the industrial procedure earlier whose thought was based on the on-line optimization strategy. In the sampling period, the current parameters in the system were used as initial state. The dynamic model is applied to predict the state response in the finite time domain. Then the open-loop optimal problem was solved according to the performance indexes. Finally, a control sequence is obtained and the first parameter in the sequence is regarded as the controlled object. In order overcome the effect of the model parameters on system control, reference [12] proposed adaptive predictive control general prediction control model based on the ideology. Reference[13] proposed robust feedback model prediction model algorithm based on the time-varying disturbance linear system in order to avoid the shortcomings of the traditional prediction model are easily affected by the system uncertainty and provided the feedback control methods for the system uncertain factors.

\section{Nonlinear time-varying system control}

The common nonlinear time-varying controller design ideology is designing the continuous controller based on the time model of the controlled objects and then discrete it. In 
order to make the performance difference between the discrete controller and continuous controller less, the sampling periods should as little as possible. The approximate discrete model of the controlled objects should be designed and then the discrete controller is designed based on the model.

1) The backstepping adaptive control

This method is a kind of construction method which uses the system construction to decompose the nonlinear system into lower order sub-systems and then constructs the stable functions or partial lyapunov functions for the sub-systems until it degraded to the whole system. The design ideology begins from the system high-order kernel. It designs the virtual control structure to ensure the system performance then gradually correct the virtual control structures until the stable controllers are obtained to fulfill the system whole areas tracking.

The reference[14] designed the self-adaptive controller for parameter output feedback discrete nonlinear system with systematic methods to avoid the lyapunov functions which are difficult to build. Compared with continuous time system, this method can only guarantee the system stability in linearly increasing without considering time-varying parameters and interferences. Thus, reference[15] proposed robust self-adaptive controller design methods which applied parameter projection and backstepping method for interference and modeling uncertainty. It provided the parameters range estimation. However there was overparameter problem in the method. Reference[16] proposes the time-varying parameters system based self-adaptive method which can avoid the over-parameter problem.

\section{2) Iteration learning control}

The iteration learning control provides better control algorithm for the nonlinear, time-varying uncertain objects. The algorithm takes advantage of the prior information to improve current control efficiency. After times of iterations, the controlled objects can obtain the expected trajectory whose precisions are as high as possible in the specific time domain[17]. The reference[18] mentions expectation trajectory variable iteration learning control method in different iteration procedures which takes advantage of the system reversely obtain the optimal input to avoid the system changes due to the trajectory changes. First, the reverse system of the dynamic system is solved. Then the wavelet is used to approach the system parameter in order to obtain the optimal approximate of the control input.

\section{FUTURE WORKS}

The time-varying systems are complex and uncertain in which there are numbers of problems.

1)Fast time-varying system control problem. Current researches are based on parts of the research results of the slow time-varying. However, for the fast time-varying, especially for high-order linear fast time-varying and nonlinear fast time-varying, the time-varying parameters in the time domain affect the existing methods in real-time identification and control, which is a hard problem now.

2)System stability analysis. The control algorithms need additional conditions and parameters while studying the time-varying system. Aiming at this types of problem, how to make the parameters and conditions not affect the system inherent time-varying feature and restrain the system as less as possible is main problem in time-varying control algorithm practical applications.

3)Real-time modeling problem. The real-time modeling of the time-varying system is the key point which determines the overall performance of the time-varying system. Current modeling uses the approximate simulations. However, the model needs to correct the control system feedback parameters real-time which make the model close to the practical system.

4)Nonlinear analysis. The differential equations of the time-varying system are hard to solve in the nonlinear conditions, even the models are not suitable. It's problematic to model the time-varying nonlinear features based on the linear model and linearize the nonlinear systems to find the linear segmentation functions to replace the nonlinear analysis reducing the nonlinear control complexity, which need further analysis and research.

\section{CONCLUSIONS}

The time-varying systems have broad application prospects and the theories become increasingly complete in researches. Current, they already form a set of control systems which integrate applicability, iterative learning and robust control. The control model applicable to the practical situations is built by stable conditions analysis which can effectively increase the efficiency of the control system and ensure the stability. However, there are still some problemssuch as data processing, applicable conditions and timevarying uncertainty. Based on the complete theoretic system, to solve the problems in the practical applications is the key in the time-varying control system development. 